\title{
Impedimetric Immunosensors Based on the Conjugated Polymer PPV
}

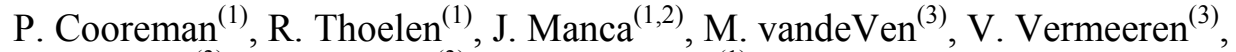 \\ L. Michiels ${ }^{(3)}$, M. Ameloot ${ }^{(3)}$, and P. Wagner ${ }^{(1)}$ \\ ${ }^{(1)}$ Institute for Materials Research, Limburgs Universitair Centrum, Wetenschapspark 1, B- \\ 3590 Diepenbeek, Belgium \\ ${ }^{(2)}$ IMEC vzw, division IMOMEC, Wetenschapspark 1, B-3590 Diepenbeek, Belgium \\ ${ }^{(3)}$ Biomedical Research Institute, Limburgs Universitair Centrum, School of Life Sciences, \\ transnationale Universiteit Limburg, B-3590 Diepenbeek, Belgium
}

\begin{abstract}
:
In the work reported here, we investigated the interaction between the semiconducting polymer MDMO-PPV and antibodies against the fluorescent dyes FITC and Cy5. The antibodies are adsorbed physically onto thin polymer films on gold electrodes, as seen in AFM images of these films. By tuning the antibody concentration, the contact angle of distilled water with the film can be made to vary between $95^{\circ}$ and $50^{\circ}$, showing that different surface densities of antibody can be obtained. That these biosensor films specifically bind their antigenic fluorescent molecules from PBS buffer solution is demonstrated by confocal fluorescence microscopy. Specific antigen-antibody recognition is demonstrated by lack of crosssensitivity between the two antibodies and their antigens. In a biosensor prototype based on differential impedance spectroscopy, these polymer films show a clear response to $1 \mathrm{ppb}$ antigen solution, with a time constant of 2 to 3 minutes.
\end{abstract}

\section{Keywords:}

immunosensors, impedance spectroscopy, conjugated polymers, immunoglobulins, DNA

\section{Introduction}

Semiconducting polymers are widely used as a base material in opto-electronic applications such as displays and solar cells (Munters et al., 2002; Martens et al., 2003). In recent years, the use of these polymers also in other applications is being investigated. An established application of an insulating polymer in biomedical research is the use of polystyrene (PS) in ELISA tests (Cantarero et al., 1980). Biomolecules are adsorbed physically to PS microwells, and via immunological or enzymatic reactions with fluorescent dyes, analyte concentrations down to 0.1 $\mathrm{fmol} / \mathrm{mL}$ can be determined. The use of a semiconducting polymer in such an experimental setup may provide the possibility of direct electronic read-out. A well- 
known example is the glucose biosensor (Hiller et al., 1996; Kros et al., 2001; Fiorito and De Toresi, 2001), based on the electron transfer that occurs during the enzymatic reduction of glucose. Research on electro-inactive reactions like antibody-antigen affinity binding is also being carried out. In the latter case, no direct amperometric signal can be detected, but changes occurring in the impedance of the bioactive film can be measured (Bender and Sadik, 1998; Farace et al., 2002; Katz and Willner, 2003; Laureyn et al., 2000; Lillie et al., 2001). The rapidity, sensitivity, and reproducibility of such measurements have recently been reported for differential impedance spectroscopy using the polymer polypyrrole (Sadik et al., 2002; Fernandez-Sanchez et al., 2004).

Our work focuses on the characterization of biologically activated polymer films to determine the parameters that influence the performance of such films in an impedimetric measurement. We present results from contact angle measurements and atomic force microscopy (AFM), which confirm that antibodies can be adsorbed to a polymer film. The binding of fluorescent antigens to antibodies is demonstrated via fluorescence microscopy, proving that the immobilized antibodies are still biologically active. Finally, we present the outcome of tests of these biofilms in an impedimetric biosensor setup.

\section{Materials}

A PPV (poly(phenylene vinylene)) derivative, MDMO-PPV, synthesized via the Gilch or sulphinyl route, is used as base polymer (Louwet et al., 1995). Monoclonal mouse antibodies (IgG) against the dyes fluorescein isothiocyanate (FITC) and Cy5 are used for recognition. The dyes FITC and Cy5 are light, fluorescent molecules with masses smaller than $1 \mathrm{kDa}$. They are conjugated, respectively, to a 250 base pair double-stranded DNA fragment, and to the protein bovine serum albumin (BSA) to obtain molecules of $154 \mathrm{kDa}$ and $77 \mathrm{kDa}$. This has the advantage that the antigens can easily be detected by AFM. Moreover, heavy molecules correspond better to the macromolecular antigens relevant in human pathology. All solutions were prepared in phosphate buffered saline (PBS).

\section{Experimental}

First, a sample series for contact angle measurements was prepared. The polymer was spin coated from chlorobenzene solution onto glass substrates $(10 \mathrm{~mm} \times 10 \mathrm{~mm})$ to form a film of $100 \mathrm{~nm}$ thickness. To minimize the transversal electrical resistance in actual biosensor measurements, a low thickness is desirable. However, the polymer should also form a closed and smooth film on the sensor electrode. Therefore, because these films were easily reproduced, and had a roughness of about $4 \AA$ (rms) on a scale of 20 sq. $\mu \mathrm{m}$ (compared to $21 \AA$ for the glass substrate), $100 \mathrm{~nm}$ thin films were used throughout this work. Then, antibodies from a neutral $10 \mathrm{mM}$ phosphate-buffered saline solution (PBS) were adsorbed onto the polymer films for 1 hour at $37^{\circ} \mathrm{C}$ in an incubation oven. Concentrations ranging between $0.01 \mathrm{pmol} / \mathrm{mL}$ and $1 \mathrm{nmol} / \mathrm{mL}$ were used, for both anti-FITC and anti-Cy5. The static contact angle was then measured with distilled water, using a Dataphysics OCA 15 Plus. AFM was used to compare polymer samples with anti-Cy5 antibodies adsorbed and Cy5-BSA bound to the antibodies with mica samples that had undergone the same treatment 
with the biomolecules. The atomic force microscope was a Park Scientific Instruments Autoprobe CP, together with the SPIP image-processing package. The fluorochromes FITC and Cy5 bound to their antibodies on the polymer film were detected with a Zeiss LSM 510 META confocal fluorescence microscope. Excitation wavelengths of $488 \mathrm{~nm}$ and $633 \mathrm{~nm}$ were used, respectively for FITC and Cy5. The wavelength resolved fluorescence intensity of the polymer was compared with that of a sample with adsorbed anti-FITC to which FITC-DNA had bound. In addition, the influence of a protein layer to prevent non-specific DNA adsorption to the polymer was investigated: samples both with and without a BSA blocking layer were exposed to FITC-DNA and measured.

After the characterization measurements, electrodes for biosensor experiments were prepared, using the same spin coat and antibody adsorption conditions. A gold contact layer was evaporated onto the glass substrate prior to spin coating. The polymer electrode, with adsorbed antibodies, was then placed into a liquid cell (see Fig. 1a). An inactive polymer film, either coated with the blocking protein BSA, or with another antibody, was also placed in the cell to allow differential measurements. The cell consists of two sample spaces connected to the liquid compartment, with counter electrodes on top of each. An inlet allows us to put the analyte solution (FITC-DNA or Cy5-DNA of $0.1 \mathrm{nmol} / \mathrm{mL}$ or less) inside both compartments. The impedance of this cell was measured with an HP 4284A Precision LCR Meter. Frequency dependent measurements ranged from $20 \mathrm{~Hz}$ to $1 \mathrm{MHz}$, and time dependent measurements were carried out at a fixed frequency of $80 \mathrm{~Hz}$. This frequency was chosen because the technique is sensitive to macroscopic charge distributions at the electrodes, which influence the impedance at lower frequencies (Lasseter et al., 2004). At frequencies around $50 \mathrm{~Hz}$ and less, however, considerable electronic noise from the environment was observed. The software package ZSimpWin from Princeton Applied Research was used to fit model equivalent circuits to the impedance data.

(a)

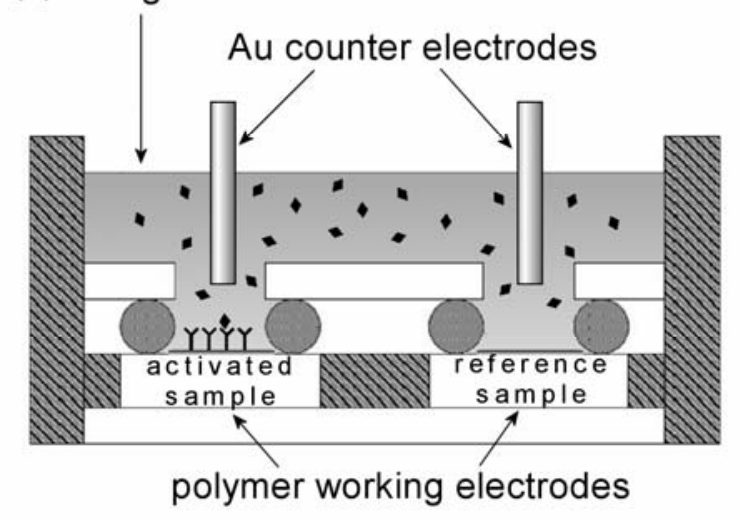

(b)

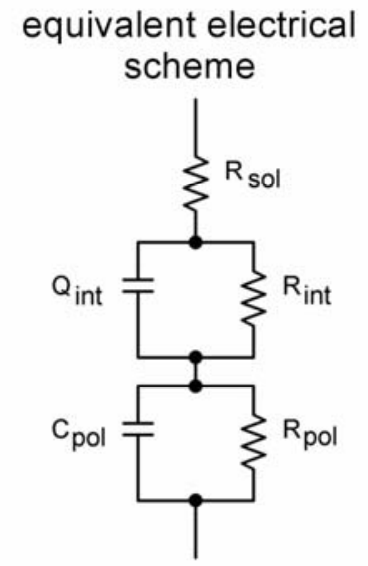

Fig. 1: (a) Schematic drawing of the (optically transparant) differential impedimetric immunosensor. The samples are in contact with the liquid compartment in a circular region with a diameter of $3 \mathrm{~mm}$, and the counter electrodes are centered $2 \mathrm{~mm}$ above each sample. (b) The equivalent electrical scheme used in theoretical fits. An explanation for the components of this circuit is given in subsection 4.4. 


\section{Results and Discussion}

\subsection{Contact Angle Measurements}

The polymer MDMO-PPV is hydrophobic, with a contact angle of $\approx 95^{\circ}$ with water. The adsorption of antibodies generates much smaller angles. When different concentrations of adsorption solutions are applied to the polymer film, the contact angle will vary between the contact angles of pure PPV and PPV maximally covered with adsorbed antibodies, respectively. In Fig. 2a, the results from two sets of samples, one with anti-FITC and one with anti-Cy5, are shown. The forms of these curves suggest that they are best described by a logistic fit (an exponential evolution between two saturation values), for which the results are also depicted in Fig. 2a. From the curves, one can see that at very low antibody concentrations there is no change in contact angle, and that high concentrations lead to a saturation value. For both types of antibodies, almost complete coverage of the polymer is obtained with adsorption concentrations of $100 \mathrm{pmol} / \mathrm{mL}$, applied for 1 hour at $37^{\circ} \mathrm{C}$. In addition, repeated washings with deionized water do not alter the contact angle. We may therefore suppose that a stable layer of biomolecules has formed on the PPV film. These findings resemble the Vroman effect (Vroman et al., 1986; Gerritsen et al., 1998)), which states that blood serum proteins adsorb to medical implants within minutes to form a (typically monomolecular) layer. A concentration of $100 \mathrm{pmol} / \mathrm{mL}$ was therefore employed for the adsorption of antibodies in further experiments, in particular, for impedimetric biosensing.

\subsection{Atomic Force Microscopy}

Various AFM measurements were performed on polymer samples with anti-Cy5 $(100 \mathrm{pmol} / \mathrm{mL})$ adsorbed onto them and Cy5-BSA $(100 \mathrm{pmol} / \mathrm{mL})$ subsequently bound to the antibodies. For reference purposes, we also used adsorbed antibodies and BSA to cleaved mica, with the advantage that the mica is atomically flat and particles can easily be detected. On the polymer surface with antibodies, a film of particles of about $20 \mathrm{~nm}$ can be observed, while on the sample with Cy5-BSA, 30 to $40 \mathrm{~nm}$ particles were seen. Good correspondence between the height profiles of the polymer and mica reference samples was observed. Thus, the particles seen in the topology of the polymer may indeed be identified with antibodies and antigens.

In the FITC-DNA interaction with the bare polymer film, the possibility of nonspecific binding of DNA to the polymer exists, and this was indeed seen by AFM. On the other hand, when a layer of native BSA (i.e., without antigen conjugation) is adsorbed to the polymer, no FITC-DNA subsequently bound to the biofilm, showing that a stable blocking layer had formed. In further impedimetric experiments however, we did not use BSA overcoating to block non-specific binding. There, we assume that the use of a sufficiently high adsorption concentration of $100 \mathrm{pmol} / \mathrm{mL}$ results in a polymer film maximally covered with $\operatorname{IgG}$, so that no direct binding to the polymer is possible.

\subsection{Confocal Fluorescence Microscopy}

The polymer MDMO-PPV has a broad absorption band, which extends into the wavelength region of FITC emission and the fluorochrome in the antibody-antigen complex lies close to the polymer surface. Therefore, the emission of FITC may be 
quenched by Förster resonance energy transfer (Berney and Danuser, 2003; Wang et al, 2004) to the absorbing groups in the polymer. In addition, the polymer itself shows a strong fluorescence, as can be seen in Fig. 2b, showing emission spectra measured for a set of MDMO-PPV samples. The FITC-DNA-loaded film shows no evidence of FITC emission around $510 \mathrm{~nm}$, indicating either complete quenching or that the FITC emission intensity is negligible as compared to the polymer

fluorescence, whose intensity is seen to peak around $625 \mathrm{~nm}$. However, it appears that there is a specific large enhancement of the polymer emission when the polymer is coated with the antibody-FITC complex (sample 2 in Fig. 2b), whereas there is only a minor enhancement when binding of the dye FITC-DNA is blocked by a coating of native BSA (sample 4 in Fig. 2b). This may be caused also by the resonance energy transfer of FITC to the polymer. In addition, the increased fluorescence of sample 3 (in Fig. 2b) as compared with samples 1 and 2 demonstrates that non-specific adsorption of $(100 \mathrm{pmol} / \mathrm{mL})$ FITC-DNA to PPV is possible and leads to a surface density of FITC comparable with that of FITC bound to its antibody. 
(a) Contact Angle Measurements
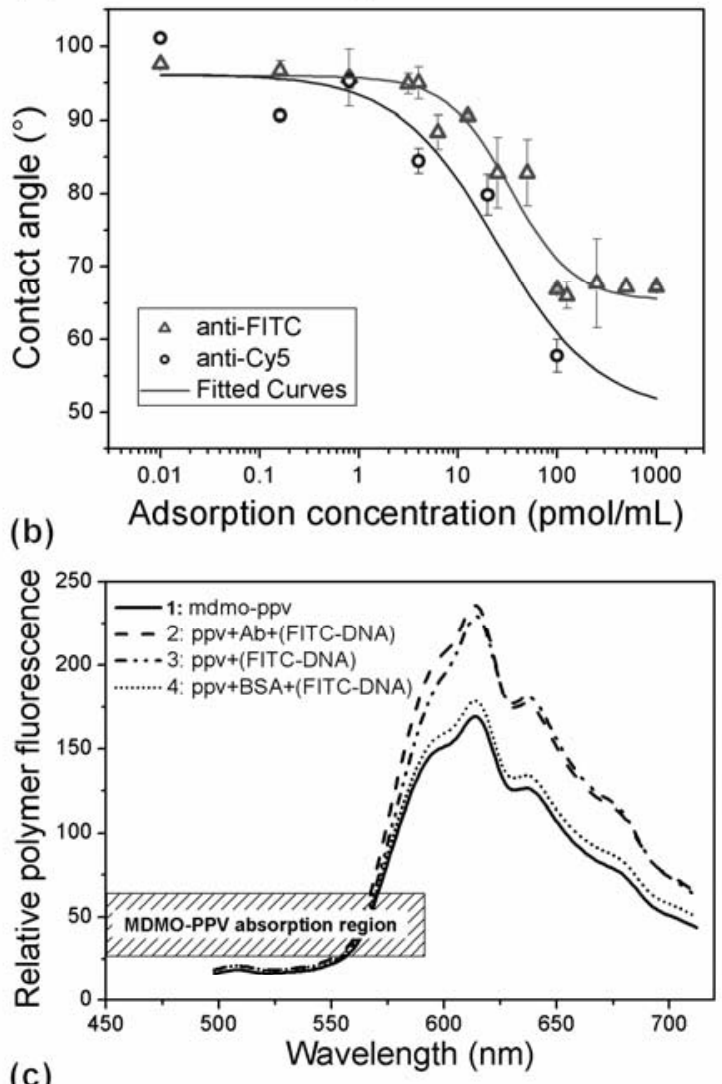

(c)

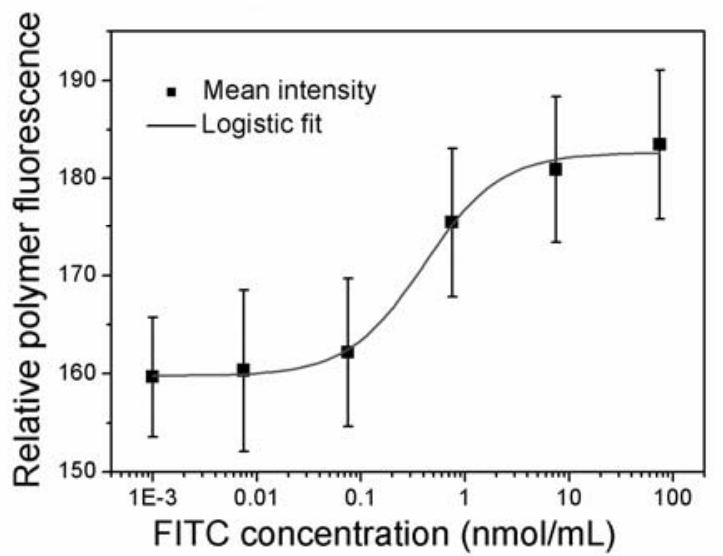

Fig. 2: (a) Contact angle measurements of water with polymer films with different concentrations of antibodies $(\mathrm{Ab})$ adsorbed onto them. Circles represent data points from anti-Cy5 adsorbed onto the polymer, and triangles from anti-FITC on the polymer. The solid lines are logistic fits of the two sets of data points, and serve as a guide to the eye (the error bars represent the standard deviation of 4 measurements). (b) Fluorescence spectra of different samples of PPV with anti-FITC and FITC-DNA due to excitation at $488 \mathrm{~nm}$. The spectral bandwidth of the detector was $10 \mathrm{~nm}$. (c) Fluorescence intensities from anti-FITC samples that have reacted to FITC at solution concentrations within the range of $1 \mathrm{pmol} / \mathrm{mL}-100 \mathrm{nmol} / \mathrm{mL}$ are shown (the errors are the standard deviation of the integrated intensity over a 1 by $1 \mathrm{~mm}$ square). The solid line is a guide to the eye. 
These findings were also examined quantitatively. If a polymer film loaded with antibodies adsorbed from a $100 \mathrm{pmol} / \mathrm{mL}$ solution is exposed to increasing concentrations of FITC, the fluorescence (integrated from $500 \mathrm{~nm}$ to $720 \mathrm{~nm}$ ) can be seen to increase in accordance with a simple saturation binding mechanism. This is shown in the sigmoidal relationship of intensity $v s$. concentration on a logarithmic scale in Fig. 2c. The trend indicated by the simple saturation curve fitted to the data points suggests that the increase in fluorescence intensity enhancement becomes appreciable at an FITC concentration of about $150 \mathrm{pmol} / \mathrm{mL}$ and is almost saturated at about $50 \mathrm{nmol} / \mathrm{mL}$. We can conclude that, despite the narrow range of increase in the enhancement of PPV fluorescence elicited by FITC binding to its adsorbed antibody, and the large standard deviation of the data points, this parameter may reliably be used to estimate the antigen (FITC) concentration in the solution over almost three orders of magnitude.

\subsection{Impedance Spectroscopy}

To test the sensitivity and cross-sensitivity of anti-FITC to Cy5-DNA and of antiCy5 to FITC-DNA, we used an anti-FITC adsorbed polymer film as an "active" electrode, and an anti-Cy5 adsorbed film as a "reference" electrode. In a first experiment, carried out at room temperature, solutions of increasing concentration of FITC-DNA were injected into the cell, and the impedance responses of both the active and the reference sample were measured. The results are shown in Fig. 3a. One can see that the anti-FITC sample responds via a decrease in impedance amplitude, and the phase angle becomes less negative, whereas the reference sample, within the noise level, shows no measurable change in phase or in impedance amplitude. The lowest concentration to which the active sample responded was $50 \mathrm{pmol} / \mathrm{mL}$ FITC-DNA, corresponding to $7.7 \mu \mathrm{g} / \mathrm{mL}$ or $0.9 \mathrm{ppb}$ in PBS. The rather small increase in amplitude observed indicates that this value is close to the detection limit of the biosensor electrode. In addition, when the concentration of FITC-DNA is increased by $16 \mathrm{pmol} / \mathrm{mL}$ and again by a further $9 \mathrm{pmol} / \mathrm{mL}$, up to a final concentration of 75 $\mathrm{pmol} / \mathrm{mL}(11.5 \mu \mathrm{g} / \mathrm{mL})$, the impedance of the active sample decreases accordingly. These results show a rather more sensitive detection limit than was observed by polymer fluorescence, and much better differential sensitivity at the low concentration range used as compared to that covered in the fluorescence method. In literature (Katz and Willner, 2003; and references therein), typically an increase in impedance amplitude is reported, contradicting our findings. However, we have used semiconductive instead of conductive electrodes, which can account for the different response to protein interactions at the electrode surface.

The same impedance setup was used in the second experiment, but with newly prepared electrode samples, and the changes in amplitude and phase of the impedance observed as a function of time are shown in Fig. 3b. In this experiment, a Cy5-DNA solution was first injected into the cell originally filled with PBS buffer, to a final concentration of $42 \mathrm{pmol} / \mathrm{mL}$. As seen in the figure, continuous drifts of both amplitude and phase measurements on both electrodes were observed prior to the addition of Cy5-DNA. After addition of this antigen, the impedance amplitude of the electrode loaded with anti-Cy5 decreased exponentially, with a time constant of 1.8 minutes (the fitted curve also displayed was obtained without taking account of the drift of the impedance amplitude and phase). The electrode loaded with anti-FITC did not respond at all to this event. Secondly, a solution of FITC-DNA was injected, to a 
final concentration of $75 \mathrm{pmol} / \mathrm{mL}$. Now the anti-Cy5 loaded electrode does not react, whilst the anti-FITC loaded sample does, with a time constant of 2.9 minutes. Compared to the measurement of Fig. 3a, the impedance amplitudes and phases respond much more to the same concentration, indicating that these electrodes have a better sensitivity. Note also that in the second measurement (Fig. 3b), the starting values of the impedance amplitude of the two different electrodes are almost the same, which was not the case in the first measurement (Fig. 3a). This implies that reproducible sample preparation and adsorption of antibodies is crucial for good sensor performance.

Frequency-dependent measurements were conducted both before and after the molecular antibody-antigen recognition. The data are shown in Fig. 3c as Nyquist plots, with the frequency ranging between $20 \mathrm{~Hz}$ and $1 \mathrm{MHz}$. It is clear that the change in impedance due to the antibody-antigen affinity binding mainly occurs at low frequencies, up to $100 \mathrm{~Hz}$. To fit these data, a simple model is used, consisting of a resistance $\mathrm{R}_{\mathrm{pol}}$ and a capacitance $\mathrm{C}_{\mathrm{pol}}$ in parallel for the bulk polymer, and a series resistance $R_{\text {sol }}$ for the buffer solution (see Fig. 1b). The interface layer with the biomolecules is modeled as a resistance $\mathrm{R}_{\text {int }}$, and a constant phase element $(\mathrm{CPE}) \mathrm{Q}_{\text {int }}$, which accounts for the capacitance-like behaviour of the interface, with influences of charge diffusion, electrode roughness, and more (Refaey, 2004). The fitting results are shown in Fig. 3c as solid lines, and the fit parameters are given in Table 1. One can see that, after antigen injection, the interface parameters have changed markedly: $\mathrm{R}_{\text {int }}$ decreases by more than $50 \%$ and $\mathrm{Q}_{\text {int }}$ increases by more than $100 \%$, of their original values. This result confirms that the measured change in impedance is indeed due to the antibody-antigen reaction at the interface layer of the electrode. However, some important remarks can be made. The fitted curves from Fig. 3c do not follow the experimental data perfectly, so the proposed equivalent circuit, which was based on simple considerations, does not completely describe the real situation. Moreover, we suppose that the drop in solvent resistance upon antigen injection occurs because the liquid space is not confined to a fixed volume, and liquid addition leads to a volume increase. 

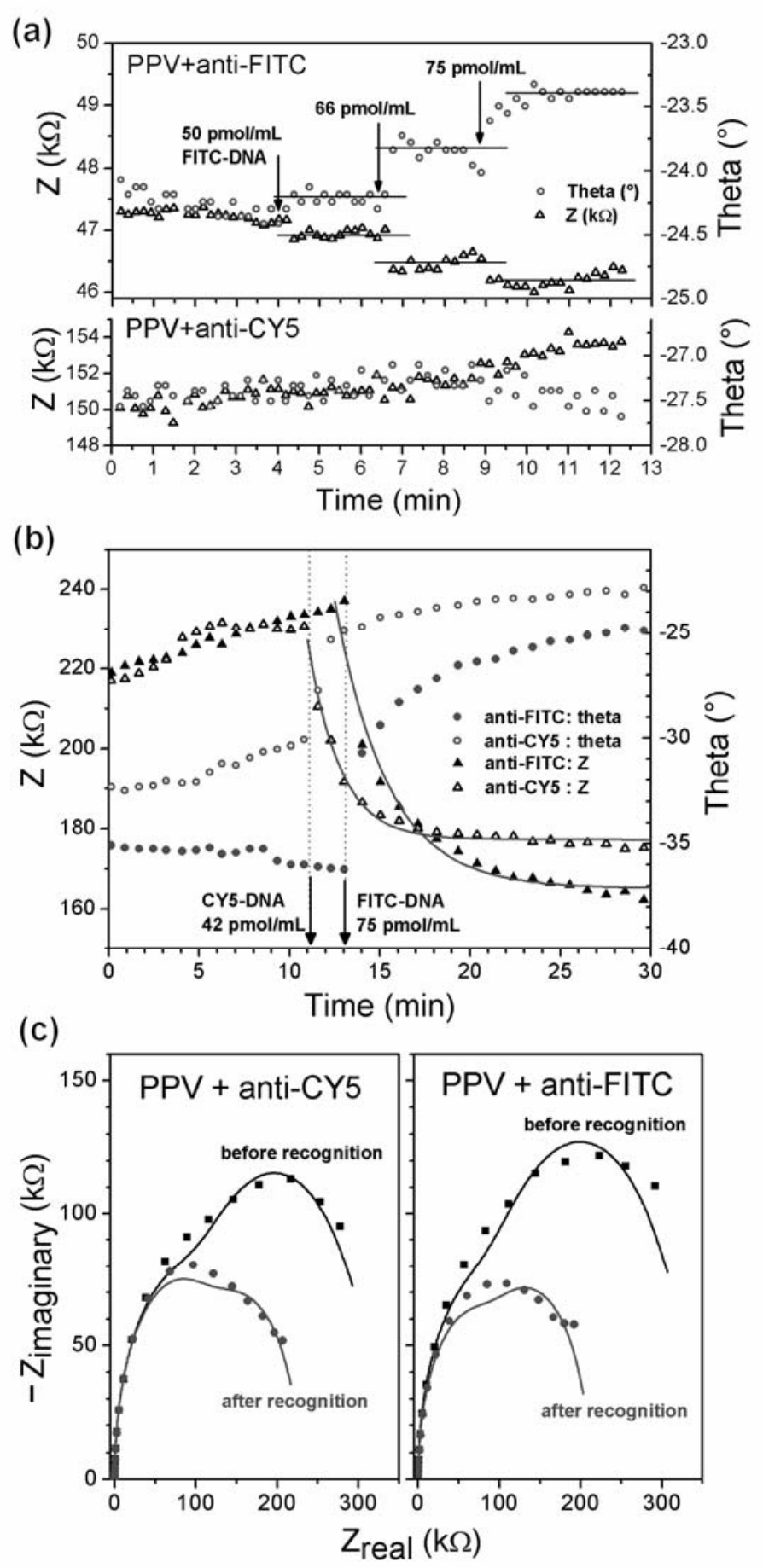

Fig. 3: Impedance amplitude $(\mathrm{Z})$ and phase (Theta) graphs for both active and reference samples, taken at $80 \mathrm{~Hz}$. Besides the observed drift, the noise level is lower than $1 \mathrm{k} \Omega$ for the impedance amplitude. In (a), three different concentrations of FITC-DNA are added, and in (b) first Cy5-DNA and then FITC-DNA are injected to the cell. Nyquist plots for the behaviour as a function of frequency $\left(Z_{\text {imaginary }} v s . Z_{\text {real }}\right)$ before and after the antigen injections are shown in (c). 
Table 1: Fitting parameters obtained from the fits of Fig. 3c (before and after recognition of Cy5 or FITC), using the model from Fig. 1b. The parameter $\alpha$ in the $5^{\text {th }}$ row is related to the CPE and is a measure of how much this element resembles a normal capacitance. The fact that $\alpha$ remains almost the same after antigen binding allows comparing the different values of $Q_{\text {int }}$ directly.

\begin{tabular}{ccccc} 
& \multicolumn{2}{c}{ anti-FITC sample } & \multicolumn{2}{c}{ anti-Cy5 sample } \\
& before & after & before & after \\
\hline $\mathrm{R}_{\text {pol }}(\mathrm{k} \Omega)$ & $134 \pm 9$ & $132 \pm 6$ & $98 \pm 5$ & $121 \pm 6$ \\
$\mathrm{C}_{\text {pol }}(\mathrm{nF})$ & $5.1 \pm 0.3$ & $4.4 \pm 0.2$ & $3.5 \pm 0.1$ & $3.0 \pm 0.1$ \\
$\mathrm{R}_{\text {int }}(\mathrm{k} \Omega)$ & $216 \pm 11$ & $96 \pm 8$ & $244 \pm 8$ & $120 \pm 7$ \\
$\mathrm{Q}_{\text {int }}\left(\mathrm{nS} . \mathrm{s}^{\alpha}\right)$ & $18 \pm 2$ & $44 \pm 7$ & $25 \pm 3$ & $56 \pm 12$ \\
$\alpha$ & $0.95 \pm 0.01$ & $0.96 \pm 0.01$ & $0.89 \pm 0.01$ & $0.87 \pm 0.02$ \\
$\mathrm{R}_{\text {sol }}(\Omega)$ & $231 \pm 5$ & $178 \pm 5$ & $208 \pm 4$ & $155 \pm 4$ \\
\hline
\end{tabular}

\section{Conclusions}

The results from AFM, fluorescence, and contact angle characterization experiments indicate that antibodies can be successfully adsorbed to the polymer MDMO-PPV. A stable, presumably monomolecular, layer of antibodies is formed, and this layer is able to bind its specific antigens. Using the enhancement of the polymer fluorescence by excitation of FITC bound to its antibody, we could relate the increase in fluorescence intensity to the concentration of the antigen FITC in solution over a wide range. A differential impedance setup was then built, using two different antibodies on the active and reference polymer electrodes. The antibody-antigen affinity reaction resulted in a fast and sensitive impedance response, even to an analyte solution concentration as low as $50 \mathrm{pmol} / \mathrm{mL}(0.9 \mathrm{ppb})$. An incremental value in concentration of $9 \mathrm{pmol} / \mathrm{mL}$ also generated a measurable impedance response. These results indicate that the adsorbed antibodies keep their biological activity. A sensitivity down to $7 \mu \mathrm{g} / \mathrm{mL}$ FITC-DNA, and a resolution of $\sim 1.4 \mu \mathrm{g} / \mathrm{mL}$ can be achieved.

\section{Acknowledgements}

This work is supported by the School for Life Sciences of the transnational University Limburg tUL, the OSTC IUAP V programme 'Quantum Size Effects in Nanostructured Materials' and the Fund for Scientific Research (FWO) Flanders (WO.035.04N 'Hybrid Systems at Nanometer Scale', and G.0252.04 'Electronic structure, morphology and charge transfer in low-bandgap polymers and polymer/carbon nanotubes hybrid films'). The antibody anti-Cy5 was a generous gift of KREATECH Biotechnology B.V., Amsterdam, The Netherlands. The technical assistance of J. Soogen and J. Baccus (Limburgs Universitair Centrum), as well as Ir. L. Naelaerts and Ing. J. Wouters (Katholieke Hogeschool Limburg) is gratefully acknowledged. Finally, special thanks go to Prof. Dr. Chris Van Haesendonck and Dr. Robert Dale for helpful discussions and English proofreading. 


\section{References}

Bender, S. and Sadik, O.A. (1998) Direct Electrochemical Immunosensor for Polychlorinated Biphenyls. Environmental Science \& Technology 32, 788797.

Berney, C. and Danuser, G. (2003) Fret or No Fret: a Quantitative Comparison. Biophysical Journal 84, 3992-4010.

Cantarero, L.A., Butler, J.E. and Osborne, J.W. (1980) The Adsorptive Characteristics of Proteins for Polystyrene and Their Significance in SolidPhase Immunoassays. Analytical Biochemistry 105, 375-382.

Farace, G., Lillie, G., Hianik, T., Payne, P. and Vadgama, P. (2002) Reagentless Biosensing Using Electrochemical Impedance Spectroscopy. Bioelectrochemistry 55, 1-3.

Fernandez-Sanchez, C., Gallardo-Soto, A.M., Rawson, K., Nilsson, O. and McNeil, C.J. (2004) Quantitative Impedimetric Immunosensor for Free and Total Prostate Specific Antigen Based on a Lateral Flow Assay Format. Electrochemistry Communications 6, 138-143.

Fiorito, P.A. and De Torresi, S.I.C. (2001) Glucose Amperometric Biosensor Based on the Co-Immobilization of Glucose Oxidase (Gox) and Ferrocene in Poly(Pyrrole) Generated From Ethanol/Water Mixtures. Journal of the Brazilian Chemical Society 12, 729-733.

Gerritsen, M., Jansen, J.A., Kros, A., Nolte, R.J.M., and Lutterman, J.A. (1998) Performance of Subcutaneously Implanted Glucose Sensors: A Review. Journal of Investigative Surgery 11, 163-174.

Hiller, M., Kranz, C., Huber, J., Bauerle, P. and Schuhmann, W. (1996) Amperometric Biosensors Produced by Immobilization of Redox Enzymes at Polythiophene-Modified Electrode Surfaces. Advanced Materials 8, 219-222.

Katz, E. and Willner, I. (2003) Probing Biomolecular Interactions at Conductive and Semiconductive Surfaces by Impedance Spectroscopy: Routes to Impedimetric Immunosensors, DNA-Sensors, and Enzyme Biosensors. Electroanalysis 15, 913-947.

Kros, A., Van Hovell, W.F.M., Sommerdijk N.A.J.M. and Nolte, R.J.M. (2001) Poly(3,4-Ethylenedioxythiophene)-Based Glucose Biosensors. Advanced Materials 13, 1555-1557.

Lasseter, T.L., Cai, W. and Hamers, R.J. (2004) Frequency-Dependent Electrical Detection of Protein Binding Events. Analyst 129, 3-8.

Laureyn, W., Nelis, D., Van Gerwen, P., Baert, K., Hermans, L., Magnee, R., Pireaux, J.J. and Maes, G. (2000) Nanoscaled Interdigitated Titanium Electrodes for Impedimetric Biosensing. Sensors and Actuators B-Chemical 68, 360-370. 
Lillie, G., Payne, P. and Vadgama, P. (2001) Electrochemical Impedance Spectroscopy as a Platform for Reagentless Bioaffinity Sensing. Sensors and Actuators B-Chemical 78, 249-256.

Louwet, F., Vanderzande, D., Gelan, J. and Mullens, J. (1995) A New Synthetic Route to a Soluble High-Molecular-Weight Precursor for Poly(PPhenylenevinylene) Derivatives. Macromolecules 28, 1330-1331.

Martens, T., D'haen, J., Munters, T., Beelen, Z., Goris, L., Manca, J., D'Olieslaeger, M., Vanderzande, D., De Schepper, L. and Andriessen, R. (2003) Disclosure of the Nanostructure of MDMO-PPV : PCBM Bulk Hetero-Junction Organic Solar Cells by a Combination of SPM and TEM. Synthetic Metals 138, 243247.

Munters, T., Martens, T., Goris, L., Vrindts, V., Manca, J., Lutsen, L., De Ceuninck, W., Vanderzande, D., De Schepper, L., Gelan, J., Sariciftci, N.S. and Brabec, C.J. (2002) A Comparison Between State-of-the-Art 'gilch' and 'sulphinyl' Synthesised MDMO-PPV/PCBM Bulk Hetero-Junction Solar Cells. Thin Solid Films 403, 247-251.

Refaey, S.A.M. (2004) Electrochemical Impedance Studies on the Electrochemical Properties of Poly(3-Methylthiophene) in Aqueous Solutions. Synthetic Metals 140, 87-94.

Sadik, O.A., Xu, H., Gheorghiu, E., Andreescu, D., Balut, C., Gheorghiu, M. and Bratu, D. (2002) Differential Impedance Spectroscopy for Monitoring Protein Immobilization and Antibody-Antigen Reactions. Analytical Chemistry 74, 3142-3150.

Vroman, L. and Adams, A.L. (1986) Adsorption of Proteins Out of Plasma and Solutions in Narrow Spaces. Journal of Colloid and Interface Science 111, 391-402.

Wang, S., Gaylord, B.S., and Bazan, G.C. (2004) Fluorescein Provides a Resonance Gate for FRET from conjugated Polymers to DNA Intercalated Dyes. Journal of the American Chemical Society 126, 5446-5451. 